\title{
Just the facts: atrial fibrillation or flutter in patients who are candidates for rhythm control
}

\author{
Ian G. Stiell ${ }^{1}$ - Elisha Targonsky ${ }^{2} \cdot$ Frank Scheuermeyer $^{3}$
}

Received: 10 December 2020 / Accepted: 23 March 2021 / Published online: 6 April 2021

(C) The Author(s), under exclusive licence to Canadian Association of Emergency Physicians (CAEP)/ Association Canadienne de Médecine d'Urgence (ACMU) 2021

\section{Clinical scenario}

A 64-year-old man presents to the emergency department (ED) with palpitations that started at rest six hours ago. He denies chest pain or shortness of breath and endorses a similar event 5 months ago that quickly resolved spontaneously. He takes perindopril for hypertension. He has a heart rate of 150 beats per minute and blood pressure $124 / 78 \mathrm{~mm} \mathrm{Hg}$. He looks well, is in no distress, has irregularly irregular but otherwise normal heart sounds, and a normal respiratory exam. His electrocardiogram (ECG) shows atrial fibrillation at a rate of 148 beats per minute with no signs of ischemia.

\section{Key clinical questions}

\section{Where rhythm control is an option for patients in acute atrial fibrillation/flutter, is pharmacological or electrical cardioversion better?}

This article is Part 2 of the two-part series that addresses the management of acute atrial fibrillation or flutter in the ED. Part 1 describes the initial approach to such patients as well as treatment of those who are not candidates for cardioversion [1]. These articles are based upon the Canadian Association of Emergency Physicians (CAEP) 2018 Acute AF/AFL Best Practices Checklist which was adapted

Ian G. Stiell

istiell@ohri.ca

1 Department of Emergency Medicine, Ottawa Hospital Research Institute, University of Ottawa, Ottawa, ON, Canada

2 Department of Emergency Medicine, North York General Hospital, Toronto, ON, Canada

3 Department of Emergency Medicine, University of British Columbia, Vancouver, BC, Canada from the Canadian Cardiovascular Society AF Guidelines [2]. The CAEP Checklist recommends restoration of sinus rhythm for ED patients presenting with acute atrial fibrillation or flutter if low risk for stroke [3]. How to identify which patients are safe for rhythm control is presented in Part 1. Recently, two Canadian randomized clinical trials have addressed the issue. Scheuermeyer compared chemicalfirst and electrical-first strategies and found both to be successful and well tolerated [4]. The RAFF2 Trial compared drug-shock (IV procainamide followed by shock if necessary) to shock-only (start with shock) strategies and found both were highly effective, rapid, and safe in restoring sinus rhythm (96.1 versus 91.7\%) with IV procainamide alone directly converting $50 \%$ of patients [5]. A shock-first strategy is associated with a shortened ED length of stay while using the drug-first strategy spares the need for resource-intensive procedural sedation protocols. Both options should be part of a shared decision-making process with patients as many have a strong preference for drugs or shock.

\section{What is the best method of achieving pharmacological cardioversion?}

Pre-treatment with rate control is not recommended as it is ineffective and delays treatment. In Canada, IV procainamide is the preferred agent for pharmacological cardioversion of atrial fibrillation or flutter in the ED as it has been widely used and is safe. Other agents have disadvantages. Amiodarone is not recommended for ED patients as it is slow to act and has low efficacy. Other options are IV vernakalant (very expensive), IV ibutilide (torsades de pointes), PO propafenone (slow) and PO flecainide (slow). IV procainamide can be safely administered in patients with a systolic $\mathrm{BP} \geq 100$ and QTc $<500 \mathrm{~ms}$ at a dose of $15 \mathrm{mg} / \mathrm{kg}$ in $500 \mathrm{ml}$ normal saline, infused over $60 \mathrm{~min}$. Patients should be monitored for hypotension and QRS complex widening $>30 \%$, and the infusion may be interrupted briefly or terminated if this occurs. 


\section{Are there tips for more effective electrical cardioversion?}

Safe electrical cardioversion requires two staff (RN/Registered Respiratory Therapist; RN/RN) in addition to the physician; while a second physician is ideal, this may not be possible in smaller hospitals. Procedural sedation should follow local guidelines, e.g. fentanyl and propofol, and should not require the presence of an anesthetist. The antero-lateral and antero-posterior pad positions are equally effective and if one does not work, try the other [5]. Avoid the sternum and breast tissue and consider applying pressure to the pads to enhance success. Start with $200 \mathrm{~J}$ of energy to avoid repeated shocks. Patients are generally ready to go home $30 \mathrm{~min}$ after successful cardioversion.

\section{Which patients require anticoagulation to decrease long-term stroke risk?}

If the patient is CHADS-65 positive, then indefinite anticoagulation is strongly recommended (Fig. 1). The CAEP Checklist encourages ED physicians to prescribe the anticoagulants themselves because early follow-up with primary care or specialists may not be feasible. If the patient has stable coronary artery disease, discontinue ASA; if already on other anti-platelet agents or PCI in past 12 months, consult cardiology.

For patients who are CHADS-65 negative, the Canadian Cardiovascular Society has made a weak recommendation based on low-quality evidence that 4 weeks of anticoagulation may be considered after acute rhythm control by drug or shock. This is controversial and the CAEP Checklist authors believe that this recommendation ought not be considered either mandatory or the standard of care, but rather an opportunity to apply patient-focused clinical judgment $[6,7]$.

Patients converting spontaneously before ED treatment should generally be prescribed oral anticoagulants according to the CHADS-65 criteria. Physicians prescribing oral anticoagulants should consider shared decision-making to include patient preferences with regards to risks and benefits.

\section{How do you prescribe oral anticoagulants?}

Direct oral anticoagulants (DOACS) are generally preferred over warfarin, but warfarin is indicated for patients with mechanical valve, moderate-severe mitral stenosis, or severe renal impairment $(\mathrm{CrCl}<30 \mathrm{ml} / \mathrm{min})$. To decrease bleeding risk, exercise caution if there is a very high fall risk; advise discontinuation of NSAIDs and heavy drinking. See Thrombosis Canada App for details;

$D O A C S$ should be avoided in pregnant or breastfeeding patients or chronic kidney disease $(\mathrm{CrCl}<30 \mathrm{ml} / \mathrm{min})$.

Dabigatran-150 mg BID; use $110 \mathrm{mg}$ BID if age $>80$ years, or $>75$ years with bleeding risk.

Rivaroxaban-20 mg daily; use $15 \mathrm{mg}$ daily if $\mathrm{CrCl}$ 30-49 $\mathrm{ml} / \mathrm{min}$.

Apixaban-5 mg BID; use $2.5 \mathrm{mg}$ BID if two of: (1) serum creatinine $>133 \mu \mathrm{mol} / \mathrm{l}$, (2) age $>80$ years, or (3) body weight $<60 \mathrm{~kg}$.

Edoxaban-60 mg daily; use $30 \mathrm{mg}$ daily if $\mathrm{CrCl}$ $30-50 \mathrm{ml} / \mathrm{min}$ or weight $<60 \mathrm{~kg}$; important drug interactions.

Warfarin can be initiated at $5 \mathrm{mg}$ daily; $1-2 \mathrm{mg}$ daily if frail, low weight, or of Asian descent. Heparin bridging not required unless there has TEE-guided cardioversion.

\section{How do you remember all this advice?}

We recommend:

- The 2018 CAEP AF/AFL Checklist, soon to be updated in 2021.

- Thrombosis Canada app for anticoagulants-https:// thrombosiscanada.ca/clinicalguides/clinical-guides-webapp/

\section{Case resolution}

The patient has a primary arrhythmia and is low risk for short-term stroke given his low CHADS-65 score and short duration of symptoms. After shared decision making, the physician and patient agree to proceed with a rhythm control strategy involving electrical cardioversion. The patient is sedated using propofol, and successfully undergoes synchronized electrical cardioversion at $200 \mathrm{~J}$ and pads placed in the A-P position. After 30 min of observation and a repeat ECG showing sinus rhythm at $84 \mathrm{bpm}$, he is discharged home. Because of his CHADS-65 score of 1, he Is initiated on apixaban $5 \mathrm{mg}$ twice daily and follow-up is arranged at the hospital's cardiology clinic within 4 weeks.

\section{Key points}

1. Cardioversion by either drug or shock are acceptable and each have their advantages 
Fig. 1 Acute atrial fibrillation or flutter patients undergoing rhythm control in the ED

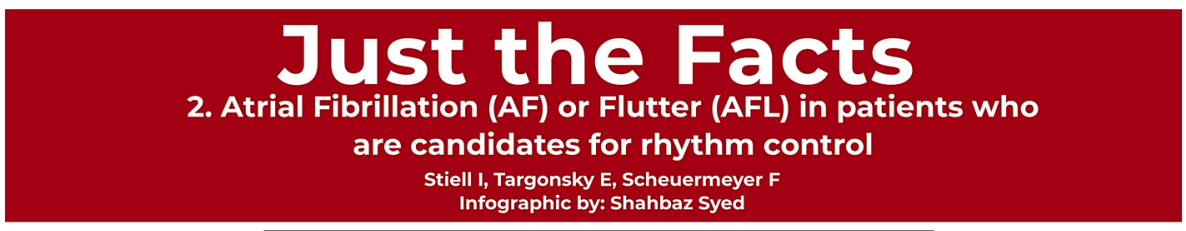

\section{Pharmacologic vs Electrical Cardioversion}

Both chemical-first and electrical=first cardioversion stratedgies have been shown to be safe and effective

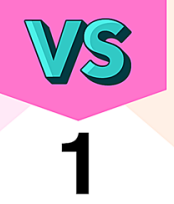

Shock first $=$ shorter ED length of stay but is more

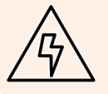

resource intensive

\section{Pharmacologic Cardioversion}

IV Procainamide is the preferred agent for pharmacologic cardioversion in the ED.
$+$

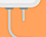

2
Procainamide $15 \mathrm{mg} / \mathrm{kg}$

IV over 60 minutes Monitor for hypotension and QRS widening (>30\%).

\section{More effective electrical cardioversion}

Antero-laterall and anteroposterior pad positions are equally effective.

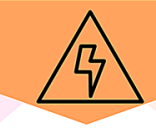

3
Start with 150-200 J to avoid

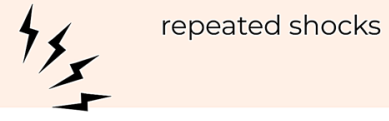

\section{Who needs immediate anticoagulation?}

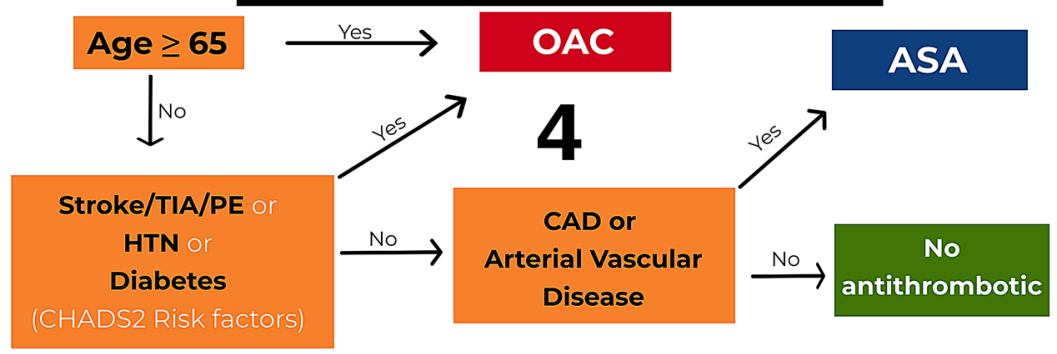

\section{Oral anticoagulants}

Warfarin is indicated if:

- Mechanical valve

- Mitral stenosis

- $\mathrm{CrCl}<30$

Find the free checklist at:

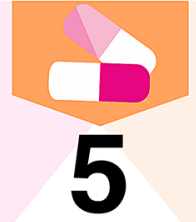

DOACs are preferred *:

- Dabigatran 150 mg BID

- Rivaroxaban 20 mg OD

- Apixaban 5 mg BID

- Edoxaban 60 mg OD

* Avoid in pregnancy/breast feeding and CKD Dose adjustments may be required 
2. CAEP recommends IV procainamide for rhythm control by drug

3. For electrical cardioversion, the antero-lateral and antero-posterior pad positions are equally effective and if one does not work, try the other

4. If the patient is CHADS-65 positive, then indefinite anticoagulation is strongly recommended

5. Direct oral anticoagulants (DOACS) are generally preferred over warfarin

\section{Declarations}

Conflict of interest The authors report no financial conflicts of interest.

\section{References}

1. Scheuermeyer FX, Targonsky E, Stiell IG. Just the facts: 1-Atrial fibrillation or flutter in patients who are candidates for rate control. CJEM. 2021. https://doi.org/10.1007/s43678-021-00116-9 (Published Online First: In Press)

2. Andrade JG, Aguilar M, Atzema C, et al. The 2020 Canadian cardiovascular society/Canadian heart rhythm society comprehensive guidelines for the management of atrial fibrillation. Can J Cardiol. 2020;36(12):1847-948. https://doi.org/10.1016/j.cjca.2020.09. 001 (Published Online First: 2020/11/17).

3. Stiell IG, Scheuermeyer FX, Vadeboncoeur A, et al. CAEP Acute atrial fibrillation/flutter best practices checklist. Can J Emerg Med. 2018;20(3):334-42.

4. Scheuermeyer FX, Andolfatto G, Christenson J, et al. A mulitcenter randomized trial to evaluate a chemical-first or electricalfirst cardioversion strategy for patients with uncomplicated acute atrial fibrillation. Acad Emerg Med. 2019;26:969-81.

5. Stiell IG, Sivilotti MLA, Taljaard M, et al. Electrical versus pharmacological cardioversion for emergency department patients with acute atrial fibrillation (RAFF2): a partial factorial randomised trial. Lancet. 2020;395(10221):339-49. https://doi. org/10.1016/s0140-6736(19)32994-0 (Published Online First: 2020/02/03).

6. Stiell IG, McMurtry MS, McRae A, et al. Safe cardioversion for patients with acute-onset atrial fibrillation and flutter: practical concerns and considerations. Can J Cardiol. 2019;35(10):1296300. https://doi.org/10.1016/j.cjca.2019.06.007 (Published Online First: 2019/09/10).

7. Stiell IG, McMurtry MS, McRae A, et al. The Canadian cardiovascular society 2018 guideline update for atrial fibrillation-a different perspective. CJEM. 2019;21(5):572-5. https://doi.org/ 10.1017/cem.2019.399 (Published Online First: 2019/09/26). 